\title{
Vertical Ridge Augmentation of the Atrophic Posterior Mandible with Sandwich Technique: Bone Block from the Chin Area versus Corticocancellous Bone Block Allograft-Clinical and Histological Prospective Randomized Controlled Study
}

\author{
Luigi Laino, ${ }^{1}$ Giovanna Iezzi, ${ }^{2}$ Adriano Piattelli, ${ }^{2}$ Lorenzo Lo Muzio, $^{1}$ and Marco Cicciù ${ }^{3}$ \\ ${ }^{1}$ Department of Clinical and Experimental Medicine, University of Foggia, FO, Italy \\ ${ }^{2}$ Department of Stomatology and Oral Science, University of Chieti, Italy \\ ${ }^{3}$ Human Pathology Department, School of Dentistry, University of Messina, Via Consolare Valeria, 98100 Messina, Italy \\ Correspondence should be addressed to Marco Cicciù; acromarco@yahoo.it
}

Received 29 March 2014; Accepted 15 April 2014; Published 29 April 2014

Academic Editor: David M. Dohan Ehrenfest

Copyright (c) 2014 Luigi Laino et al. This is an open access article distributed under the Creative Commons Attribution License, which permits unrestricted use, distribution, and reproduction in any medium, provided the original work is properly cited.

\begin{abstract}
The aim of the present study is to compare the histological aspects of bone formation in atrophic posterior mandibles augmented by autologous bone block from chin area with corticocancellous bone block allograft used as inlays with the sandwich technique. Materials and Methods. Sixteen patients with bilateral partial edentulism in the posterior mandible were selected. The residual bone height, preliminarily measured by computed tomography scans, ranged between 5 and $7 \mathrm{~mm}$ from the inferior alveolar nerve. All patients required regeneration procedure with autologous bone block from chin area (control group) versus bone block allograft Puros (Zimmer Dental, 1900 Aston Avenue, Carlsbad, CA, USA) (test group). Histological and histomorphometric samples were collected at the time of implant positioning in order to analyze the percentage of newly formed bone, the residual graft material, and marrow spaces/soft tissue. Results. No statistically significant differences between the two groups were found regarding the percentage of newly formed bone. The percentage of residual grafted material was significantly higher in the test group, whilst the percentage of marrow spaces was higher in control group. Conclusions. In conclusion, both procedures supported good results, although the use of bone blocks allograft was less invasive and preferable than harvesting bone from the mental symphysis.
\end{abstract}

\section{Introduction}

The rehabilitation of posterior mandible with dental implants represents today a hard challenge for clinicians due to the lack of supporting bone. The alveolar nerve presence and lifting and the gradual vertical and horizontal resorption of the mandibular bone crest in both partially and totally edentulous patients can be treated by several prosthetic and surgical options [1-5]. Patients can be rehabilitated with conventional partial removable dentures, but often this treatment does not meet the expectations of the patients. Regarding implant supported treatment options, vertical ridge augmentation, surgical displacement of the inferior alveolar nerve, and, finally, the placement of short implants ( $8 \mathrm{~mm}$ or less) could be necessary for the correction of the atrophic posterior mandible. The use of short implants represents a simpler and faster alternative to the augmentation procedure, even if in some "critical cases" the residual bone crest above the inferior alveolar nerve is only 5-7 $\mathrm{mm}$ in height, and therefore the surgical augmentation treatment is mandatory. Indeed, the displacement of the alveolar nerve is technically tough, and this procedure may be associated with certain degree of permanent loss of nerve sensitivity [1,6-9]. Different surgical techniques are currently being used to augment the posterior mandible: guided bone regeneration (GBR) and alveolar distraction osteogenesis onlay bone grafting; however, only few of these have been tested in randomized clinical trial (RCT) [10,11]. Several surgical bone augmentation techniques are related to an unpredictable resorption of the grafted material. Vascularity seems to be the main factor 
in determining whether such a graft can be maintained in situ. Traditional distraction osteogenesis aims to maintain the majority of the vascularity to the transported bone segment. The drawbacks of distraction osteogenesis include patient cooperation, technique sensitivity, and the possibility of a second surgery to remove the device $[1,3,7]$.

Another possible approach is to use an interpositional bone graft $[1,4,8,11]$. The rationale of the interpositional techniques is based on the theory that biomaterial placed between 2 pieces of pedicled bone with internal cancellous bone will undergo rapid and complete healing and graft incorporation with a lower percentage of resorption. The sandwich osteotomy allows for the positioning of the graft in a well-delimited area as well as offering adequate blood supply to maintain new bone growth. This procedure enables the simultaneous correction of the sagittal intermaxillary relationship and the vertical dimension. This technique has been used in a variety of maxillary areas including both the anterior and posterior mandible and maxilla. When performing the sandwich osteotomy in the posterior mandible, great surgical precision is required to avoid damage to the inferior alveolar nerve. For these reasons and for the few results available in the literature, it is necessary to carry out further research to validate the predictability of this regenerative technique $[1,3,8-11]$.

The aim of the present study is to compare the histological aspects of bone formation in atrophic posterior mandibles augmented by autologous bone block from chin area (control group) to Puros bone block allograft (test group) used as inlays with the sandwich technique.

\section{Materials and Methods}

Between November and April 2010, nineteen patients with bilateral partial edentulism in the posterior mandible were selected for the present study. They all showed a residual bone height ranging between 5 and $7 \mathrm{~mm}$ from the inferior alveolar nerve, which was firstly measured by computed tomography scans. All patients required the placement of at least 3 implants. The protocol of the study was approved by the Ethical Committee of the Second University of Naples, Naples, Italy, and all the patients signed a written informed consent form. All patients were treated in the Department of Oral and Maxillofacial Surgery, Second University of Naples, Naples, Italy. Exclusion criteria were (1) general contraindications to implant surgery, (2) irradiation, chemotherapy, or immunosuppressive therapy over the past 5 years, (3) poor oral hygiene and motivation, (4) active periodontitis, (5) uncontrolled diabetes, (6) pregnancy or lactation, (7) substance abusers, (8) smoking more than 10 cigarettes per day, (9) psychiatric problems or unrealistic expectations, (10) acute infection in the area intended for implant placement, (11) positive to HIV and hepatitis B and C, (12) autoimmune diseases such as rheumatoid arthritis, systemic lupus erythematosus, scleroderma, Sjogren's syndrome, and dermatomyositis/polymyositis, (13) treated or under treatment with intravenous aminobisphosphonates, (14) previously subjected to reconstructive procedures of the posterior mandible, and (15) under chronic treatment with steroids or nonsteroidal anti-inflammatory drugs. Twelve patients were considered eligible and were enrolled in the trial (mean age was 57 years, 9 females and 3 males).

2.1. Augmentation Procedure. Two weeks before bone augmentation and implant placement, all patients underwent oral hygiene instructions and professional debridement, when necessary. On the day of the augmentation procedure, the envelopes containing the randomized codes were opened. All patients received antibiotics prior to the surgery. Antimicrobial prophylaxis was obtained with the use of $1 \mathrm{gr}$ of amoxicillin + clavulanic acid (Augmentin, GlaxoSmithKline, Brentford, Middlesex, UK) (or erythromycin $500 \mathrm{mg}$ if allergic to penicillin), starting one day before surgery and for the following 4 days. All patients were treated under local anesthesia with intravenous sedation. A paracrestal incision was made through the buccal mucosa respecting the emergence of the mental nerve, and, as the full thickness flap was retracted, tension on the mental nerve was carefully avoided. The horizontal osteotomy was made at $4 \mathrm{~mm}$ from the mandibular canal using conventional surgical micromotor. Two oblique cuts were made in the coronal third of the mandibular bone with the mesial cut at least $2 \mathrm{~mm}$ distal to the last tooth in the arch Figures 1(a), 1(b), and 1(c). The osteotomies were completed with the use of bone chisels. The height of the osteotomized segment was at least $3 \mathrm{~mm}$ to allow the insertion of a stabilizing screw without risking the fracture of the distracted bone segment. The segment was elevated preserving the lingual periosteum, and according to the outcome of the randomization, the graft materials were modelled to the desired height and shape to fill the site and interposed between the raised fragment and the mandibular basal bone. Titanium miniplates and miniscrews (Gebruder Martin GmbH \& Co., KG, Tuttlingen, Germany) were used to fix the osteotomized crestal bone to the basal bone. The grafted area was covered with a resorbable barrier of pericardium (Copios Pericardium Membrane Zimmer Dental, Switzerland) Figures 2(a), 2(b), and 2(c). Periosteal incisions were made to release the flaps coronally as needed and were sutured with Vicryl 5.0 sutures until the incisions were perfectly sealed. Patients were instructed to use Corsodyl gel $1 \%$ twice a day for 2 weeks and then 0.2 chlorhexidine mouthwashes twice a day for up to the second month, to avoid brushing and trauma on the surgical sites. Removable prostheses were not allowed. Patients were seen after 10 days for follow-up examinations and sutures removal. Patients were recalled for additional postoperative check-ups 1, 2, and 4 months after the augmentation procedure. Four months after augmentation, a CT scan was taken to plan implant placement.

2.2. Implant Placement. Six months after the augmentation procedure, at the moment of dental implant surgery, miniplates were removed and the bone core biopsies were retrieved by using $2.9 \mathrm{~mm}$ diameter trephine bur (Komet 227b, Italy), and 72 implants (Spline Zimmer Dental, Switzerland) were inserted in situ, as shown in Figure 3. Drills with 


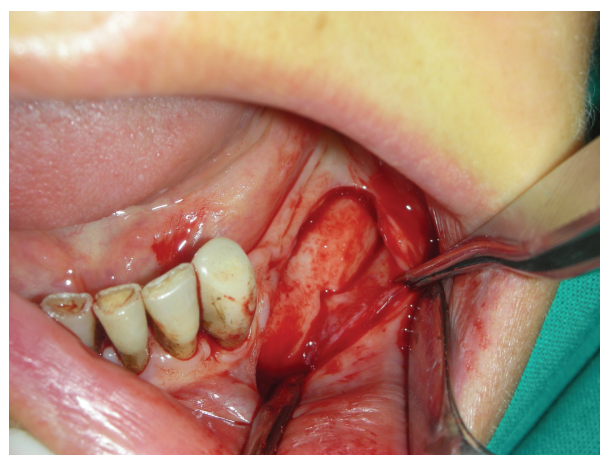

(a)

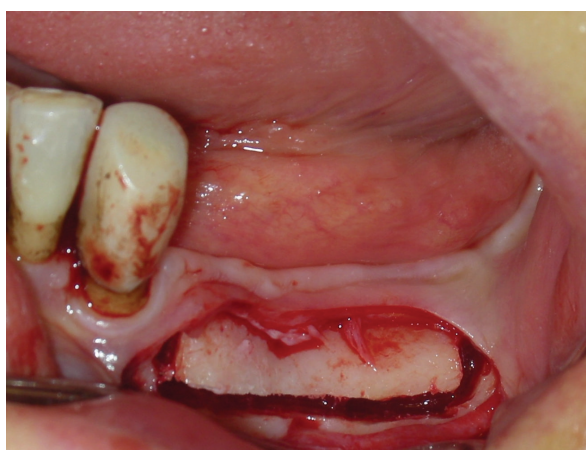

(b)

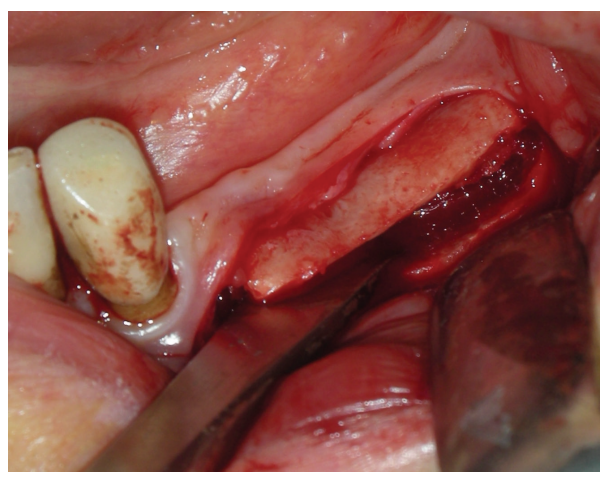

(c)

Figure 1: Sample of the two oblique cuts performed in the coronal third portion of the mandibular bone with the mesial cut at least $2 \mathrm{~mm}$ distal to the last tooth in the arch $(a, b, c)$.

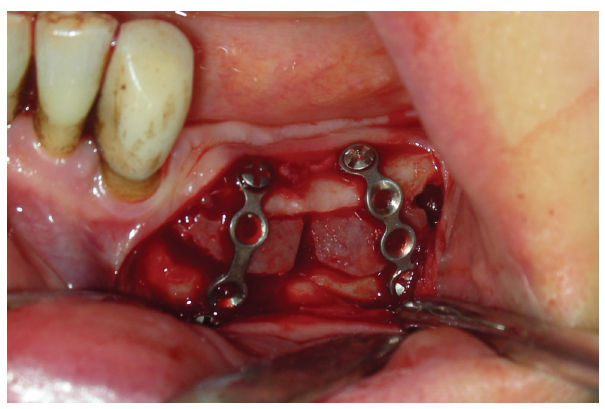

(a)

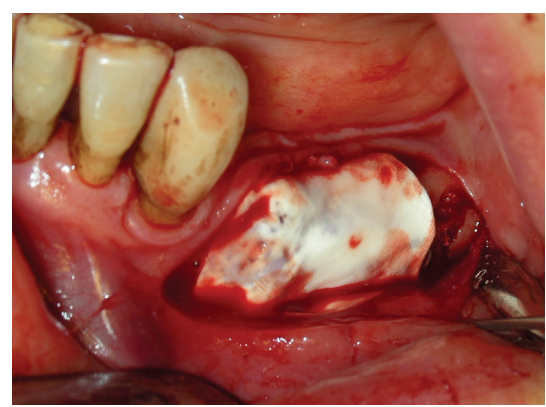

(b)

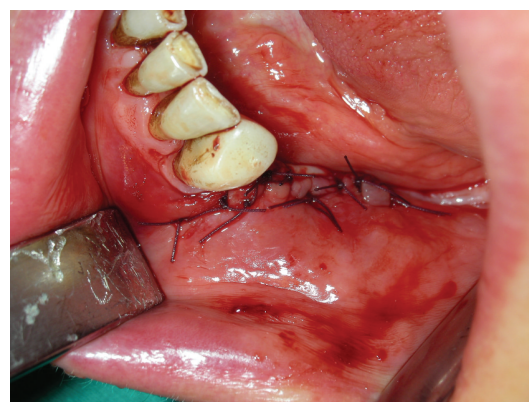

(c)

FIgURE 2: Sample of the grafted area covered with a resorbable barrier of pericardium $(a, b, c)$.

increasing diameters were used to prepare the implant sites. The surgical unit was settled with a torque of $25 \mathrm{Ncm}$. After the dental implant placement, the cover screws were placed and the flap closure was obtained with Vicryl 4.0. Patients were instructed to use $0.2 \%$ chlorhexidine mouthwash for 1 min twice a day for 2 weeks, to have a soft diet for 1 week, and to avoid brushing and trauma on the surgical sites. No removable prosthesis was allowed. Sutures were removed after 10 days.

2.3. Histological Procedure. Bone cores were retrieved, immediately stored in $10 \%$ buffered formalin, and processed to obtain thin ground sections using the Precise 1 Automated
System (Assing, Rome, Italy). The specimens were dehydrated in a graded series of ethanol rinses and embedded in a glycol methacrylate resin (Technovit 7200 VLC, Kulzer, Wehrheim, Germany). After polymerization, the specimens were sectioned, along their longitudinal axis, with a highprecision diamond disc at about $150 \mu \mathrm{m}$ and ground down to about $30 \mu \mathrm{m}$ with a specially designed grinding machine. The slides were stained with acid fuchsin and toluidine blue and examined in normal transmitted light under a Leitz Laborlux microscope (Laborlux S, Leitz, Wetzlar, Germany). Histomorphometry of the percentages of newly formed bone, residual grafted material, and marrow spaces was carried out using a light microscope (Laborlux S, Leitz, Wetzlar, Germany) connected to a high resolution video camera 


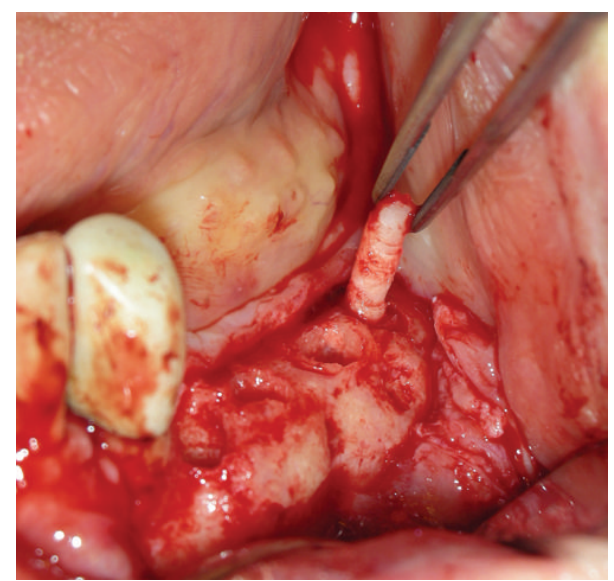

FIGURE 3: Sample of the bone core biopsies that were retrieved by using $2.9 \mathrm{~mm}$ diameter trephine bur.

(3CCD, JVC KY-F55B, JVC, Yokohama, Japan) and interfaced to a monitor and PC. This optical system was associated with a digitizing pad (Matrix Vision $\mathrm{GmbH}$, Oppenweiler, Germany) and a histometry software package with image capturing capabilities (Image-Pro Plus 4.5, Media Cybernetics Inc., Immagini \& Computer Snc, Milan, Italy). The same investigator made all the measurements.

2.4. Statistical Analysis. Data were evaluated by the ShapiroWilk test. All the data are presented as mean $+/-$ standard deviations (SD); statistically significant differences were accepted as $P<0.05$.

\section{Results}

The failures and complications that occurred during the entire study period were limited. In one patient treated with the Puros bone block, exposure of a titanium plate 2 months after surgery occurred; it was treated by removing the plaque, and then a satisfactory healing was achieved. In two patients treated with autologous bone from mental symphysis, a temporary paresthesia of the anterior region of the mandible was appreciated and treated by drug solution, Dobetin $5000 \mathrm{mcg} 1$ time per day for 1 week and 3 doses in the second week.

\subsection{Histological Results}

3.1.1. Control Group. In the control group, a significant amount of grafted bone, almost completely surrounded by newly formed bone, was observed (Figure 4). The autologous grafted bone showed irregularly shaped margins, probably due to the remodeling process. The demarcation line (cementing line) between grafted bone and newly formed bone was evident (Figure 5). In some areas, bone remodeling was conceivable with a rim of osteoblasts depositing osteoid matrix (Figure 6). Osteons in the vicinity of grafted bone could be observed (Figure 7). No signs of inflammatory infiltrate were present.

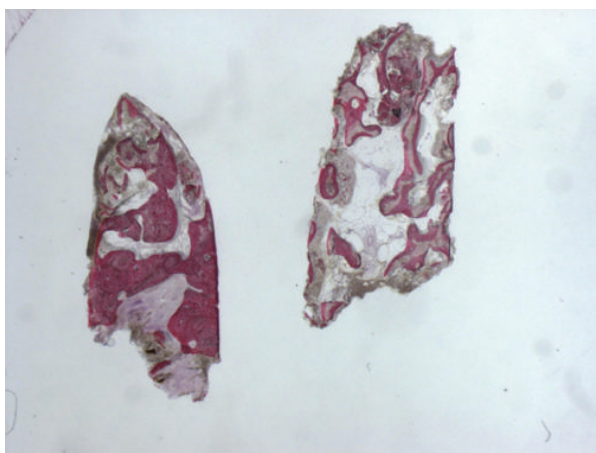

FIGURE 4: Grafted bone, almost completely surrounded by newly formed bone can be observed. Acid fuchsin-toluidine blue; original magnification $60 \mathrm{x}$.

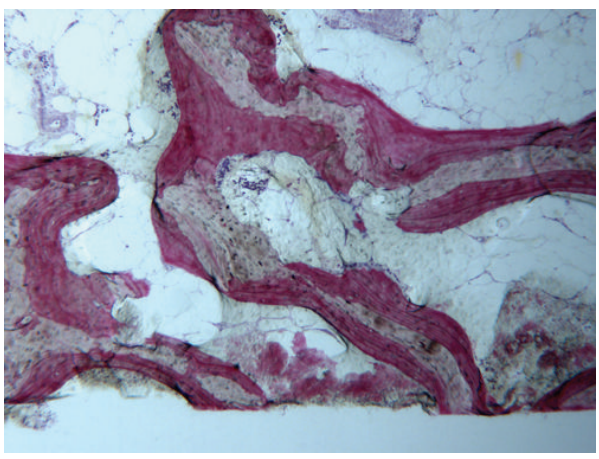

FIGURE 5: The autogenous bone block presents marked staining differences from the host trabecular bone and specifically, it shows a lower affinity for the stains. The block is surrounded by newly formed bone. Acid fuchsin-toluidine blue; original magnification $40 \mathrm{x}$.

3.1.2. Test Group. In all the analyzed samples, a good amount of newly formed bone could be observed (Figure 8). A tight contact between the grafted material and the regenerated bone, without any interposition of fibrous tissue, was found (Figure 9). The newly formed bone had a high affinity for dyes and was acid fuchsine positive; therefore, a highly stained line was observed at the grafting material-new bone interface. In many fields, it was possible to observe the presence of large osteocytes lacunae in contact with the grafted material (Figure 10). Some trabeculae of grafted material were bridged by newly formed bone, which was observed both in the inner and outer portions of some biomaterial particles (Figure 11). Marrow stromal cells and blood vessels were found inside the marrow spaces. In some fields, there was a modest amount of inflammatory infiltrate. No osteoclasts were observed around the graft particles.

The histomorphometric results are summarized in Tables 1,2 , and 3 .

There was no statistically significant difference between the two groups in terms of amount of new bone, $31.47 \pm 2.2$ versus $30.6 \pm 3.7 \%$ ( $P=0.5362$ has been recorded, while there was a statistically significant difference in the percentage of residual grafted material higher in test group (Table 2). 


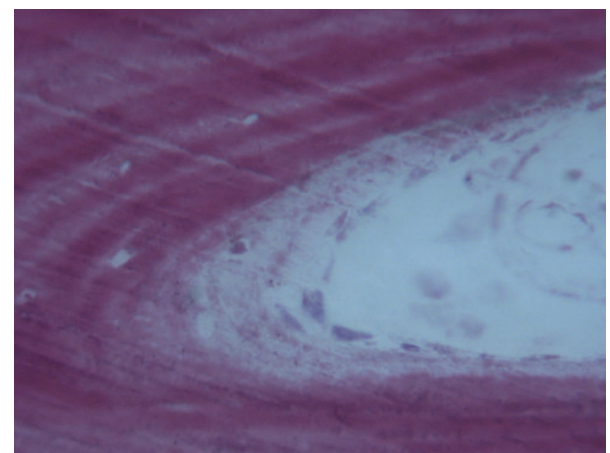

FIGURE 6: A rim of osteoblasts depositing osteoid matrix is evident. Acid fuchsin-toluidine blue; original magnification 200x.

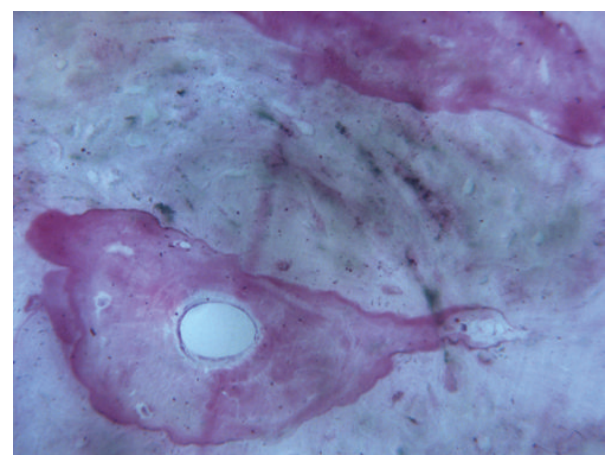

FIGURE 7: An osteon in the vicinity of grafted bone can be seen. Acid fuchsin-toluidine blue; original magnification 200x.

TABLE 1

\begin{tabular}{lccccc}
\hline Group & Obs. & Mean & Std. error & Std. deviation & 95\% conf. I. \\
\hline Group Ctrl & \multirow{2}{*}{12} & \multirow{2}{*}{31.47} & \multirow{2}{*}{7155495} & 2.262 .766 & $\begin{array}{c}29.85131 \\
33.08868\end{array}$ \\
\hline Group test & \multirow{2}{*}{12} & \multirow{2}{*}{30.6} & \multirow{2}{*}{117.936} & \multirow{2}{*}{3.729 .462} & $\begin{array}{c}27.9321 \\
33.2679\end{array}$
\end{tabular}

Newly formed bone, $t$ value $=0.6307$, and $P$ value $=0.5362$. There is no statistically significant difference.

TABLE 2

\begin{tabular}{lccccc}
\hline Group & Obs. & Mean & Std. error & Std. deviation & 95\% conf. I. \\
\hline Group Ctrl & \multirow{2}{*}{12} & \multirow{2}{*}{19.56} & \multirow{2}{*}{1.320 .959} & 417.724 & $\begin{array}{c}16.57178 \\
22.54822\end{array}$ \\
\hline Group test & \multirow{2}{*}{12} & \multirow{2}{*}{28.9} & \multirow{2}{*}{1.600 .069} & 5.059 .864 & $\begin{array}{c}25.28039 \\
32.51961\end{array}$
\end{tabular}

Residual graft material, $t$ value $=-4.5015$, and $P$ value $=0.0003$. There is statistically significant difference.

\section{Discussion}

This study is designed to evaluate how a bone substitute material may offer some advantages in the place of autogenous bone grafts harvested from the mental symphysis in the treatment of atrophic posterior mandibles. Moreover, the authors proposed a novel technique for localized vertical bone augmentation using an interpositional bone block
FIGURE 8: A good amount of newly formed bone can be observed. Acid fuchsin-toluidine blue; original magnification 6x.

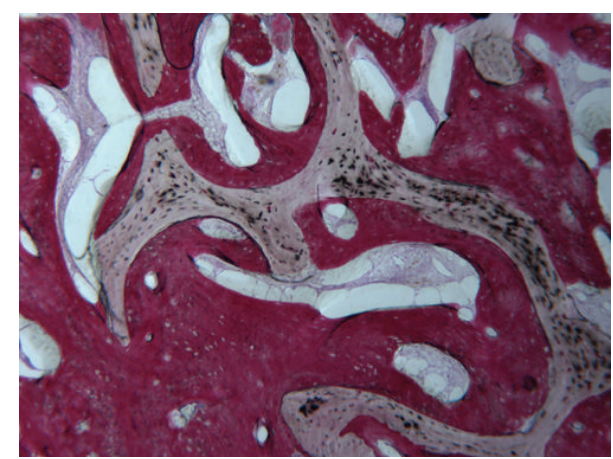

FIGURE 9: The bovine bone block is surrounded by newly formed bone. A tight contact between the grafted material and the regenerated bone without any interposition of fibrous tissue can be observed. Acid fuchsin-toluidine blue; original magnification 40x.

TABLE 3

\begin{tabular}{lccccc}
\hline Group & Obs & Mean & Std. error & Std. deviation & 95\% conf. I. \\
\hline Group Ctrl & 12 & \multirow{2}{*}{48.97} & \multirow{2}{*}{1.878 .241} & 5.939 .519 & $\begin{array}{l}44.72112 \\
53.21888\end{array}$ \\
\hline Grouptest & \multirow{2}{*}{12} & \multirow{2}{*}{41.28} & \multirow{2}{*}{1.888 .491} & 5.971 .934 & $\begin{array}{c}41.84335 \\
48.40665\end{array}$ \\
\hline
\end{tabular}

Marrow space, $t$ value $=2.8872$, and $P$ value $=0.0098$. There is statistically significant difference.

representing a valuable and predictable surgical alternative technique for posterior mandible atrophic ridge.

In 2006, Jensen retrospectively evaluated the crestal stability of alveolar augmentation using an interpositional bone graft for dental implant restorations and found a good stability after 4-year follow-up. In 2008, Felice et al. produced a series of clinical investigations for analyzing the effectiveness of this technique in relation to the use of biomaterial, and also in this case the results appeared satisfactory [12-15].

Another main aspect is the choice of the graft material to be used. In 2009, Felice et al. performed a randomized controlled clinical trial to evaluate two different kinds of graft materials: bone form iliac crest and bovine anorganic bone. There were ten selected partially edentulous patients having $5-7 \mathrm{~mm}$ of residual crystal height above the mandibular canal. Four months after bone grafting, a bone core was 


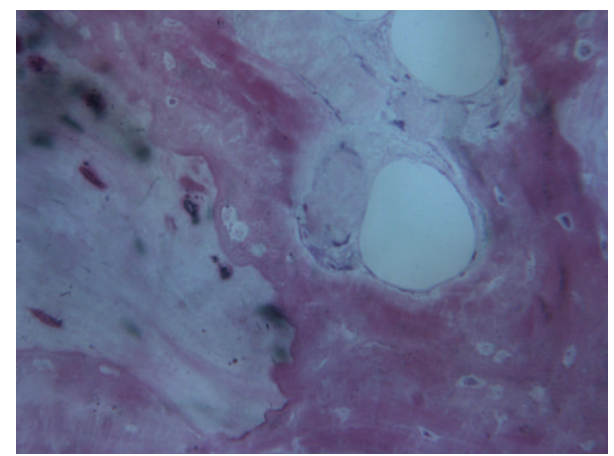

FIGURE 10: Large osteocyte lacunae in contact with the grafted material are present. Acid fuchsin-toluidine blue; original magnification 200x.

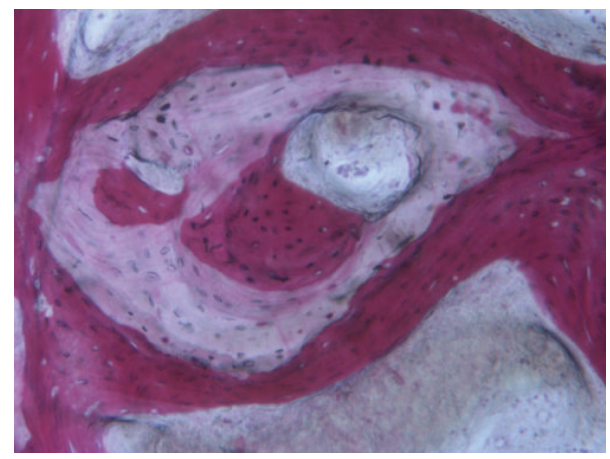

FIGURE 11: New bone can be seen in the inner and outer portions of a residual grafted particle. Acid fuchsin-toluidine blue; original magnification 100x.

retrieved from each side using a $3 \mathrm{~mm}$ external diameter trephine for the histological evaluation. The histomorphometric data showed the only statistically significant difference in the mean percentage of residual graft (between $10 \%$ and $13 \%, P$ values between 0.008 and 0.009 ) that was greater in the Bio-Oss group, while there were no statistically significant differences in the percentage of newly formed bone and in the marrow space between the two groups. Also the clinical outcomes present in the literature are very interesting $[6,12$, 14-18]. In 2006, Jensen published a retrospective study to evaluate the crystal stability of alveolar augmentation using an interpositional bone graft for dental implant restorations. Eight patients with 10 graft sites were followed from 1 to 4 years with panographic evaluation to determine if dimension changes of the alveolar graft sites had occurred. The author described a little loss of crystal height and 20 of the 22 implants confirmed high stability at the follow up evaluation. These results were later confirmed by various studies conducted by the group of Felice et al. $(2008,2009)$ and the results of this study confirmed the possibility of considered this surgical technique like predictable one $[2,4,7,12,16,18]$.

The sandwich osteotomy can be considered an alternative to other bone augmentation techniques presented in the recent literature. Although one study determined that interpositional bone grafting and alveolar distraction yielded statistically similar results in regard to buccolingual width, it later stated that bone grafting may form wider bone. No mention was made of the advantage gained with the copious blood supply by the sandwich osteotomy technique [14-19].

Other studies have shown that fewer cases of dehiscence were observed with the sandwich osteotomy than with techniques using only graft or titanium mesh. Laviv et al. recently reported that in 10 patients treated with a similar technique, vertical gain ranged from 3 to $6 \mathrm{~mm}$ over a 4 year period [16]. Authors stated that efforts to displace the segment greater than $5 \mathrm{~mm}$ "may not only risk the potential for vascular embarrassment by detaching periosteal blood supply, but also can excessively rotate the segment palatally, compromising aesthetic gingival projection." In the anterior maxilla, it has been shown that one of the disadvantages could be the reduced extensibility of the palatal mucoperiosteum that does not allow a vertical increase of more than $10 \mathrm{~mm}$ to be done $[6,18-20]$. In a letter to the editor, Robiony et al. suggest that the vertical movement could be extended more than the $10 \mathrm{~mm}$ proposed by Jensen, but only in the canine and premolar zones. They described their experience with 25 patients and demonstrated that the technique can be successful without compromising vascular supply and esthetics [20-24]. All those studies clearly demonstrated, even with some limitations, how the use of this technique, by expert surgeons, might be safe and predictable giving less discomfort for the patients [14, 22, 25].

In the present study, in order to offer our patients a less invasive surgery, a biomaterial has been compared to autologous bone and the difference in newly formed bone percentages was not statistically significant. During the histomorphometric evaluation, the percentage of newly formed bone was found to be lower in the test group (28.9/19.5); this meant a slower integration of the grafted material, which is not clinically appreciable; therefore, the use of autologous bone blocks does not seem to provide particular advantages.

\section{Conclusion}

The results of the present investigation encourage further studies on the present topic. However, other clinical trials are needed, with a greater number of patients. The results reported in the literature and this preliminary study underline how the interposition technique seems to be a valid therapeutic option in the treatment of vertical atrophy of the posterior mandible. Both graft materials gave good results in relation to this type of surgical technique; the use of Puros bone block allograft represents a less invasive alternative for the patients. In the future, it would be interesting to compare this technique with short implants and to record those results over the long term.

\section{Conflict of Interests}

As the corresponding author, Marco Cicciù declares that all the authors have no conflict of interests regarding the devices used for this study and that the current research is not influenced by any secondary interests, such as financial gain. 


\section{References}

[1] F. D. Das Neves, D. Fones, S. R. Bernardes, C. J. do Prado, and A. J. F. Neto, "Short implants-an analysis of longitudinal studies," The International Journal of Oral \& Maxillofacial Implants, vol. 21, no. 1, pp. 86-93, 2006.

[2] H. Li, C. R. Zhou, M. Y. Zhu, J. H. Tian, and J. H. Rong, "Preparation and characterization of homogeneous hydroxyapatite/ chitosan composite scaffolds via in-situ hydration," Journal of Biomaterial and Nanobiotechnology, vol. 1, pp. 42-49, 2010.

[3] K. A. Zimmermann, J. M. Leblanc, K. T. Sheets, R. W. Fox, and P. Gatenholm, "Biomimetic design of a bacterial cellulose/hydroxyapatite nanocomposite for bone healing applications," Materials Science and Engineering C, vol. 31, no. 1, pp. 43-49, 2011.

[4] B. Rosenquist, "Implant placement in combi- nation with nerve transpositioning: experiences with the first 100 cases," The International Journal of Oral \& Maxillofacial Implants, vol. 9, pp. 522-531, 1994.

[5] A. S. Herford, R. Tandon, T. W. Stevens, E. Stoffella, and M. Cicciù, "Immediate distraction osteogenesis: the sandwich technique in combination with rhBMP-2 for anterior maxillary and mandibular defects," Journal of Craniofacial Surgery, vol. 24, no. 4, pp. 1383-1387, 2013.

[6] M. Chiapasco, M. Zaniboni, and L. Rimondini, "Autogenous onlay bone grafts vs. alveolar distraction osteogenesis for the correction of vertically deficient edentulous ridges: a 2-4-year prospective study on humans," Clinical Oral Implants Research, vol. 18, no. 4, pp. 432-440, 2007.

[7] A. S. Herford, M. Lu, L. Akin, and M. Cicciù, "Evaluation of a porcine matrix with and without platelet-derived growth factor for bone graft coverage in pigs," The International Journal of Oral \& Maxillofacial Implants, vol. 27, no. 6, pp. 1351-1358, 2012.

[8] M. Merli, F. Bernardelli, and M. Esposito, "Horizontal and vertical ridge augmentation: a novel approach using osteosynthesis microplates, bone grafts, and resorbable barriers," International Journal of Periodontics and Restorative Dentistry, vol. 26, no. 6, pp. 581-587, 2006.

[9] M. Simion, S. A. Jovanovic, C. Tinti, and S. P. Benfenati, "Longterm evaluation of osseointegrated implants inserted at the time or after vertical ridge augmentation: a retrospective study on I23 implants with 1-5 year follow-up," Clinical Oral Implants Research, vol. 12, no. 1, pp. 35-45, 2001.

[10] J. H. Fu, T. J. Oh, E. Benavides, I. Rudek, and H. L. Wang, "A randomized clinical trial evaluating the efficacy of the sandwich bone augmentation technique in increasing buccal bone thickness during implant placement surgery: I. Clinical and radiographic parameters," Clinical Oral Implants Research, vol. 25, no. 4, pp. 458-467.

[11] S.-H. Park, K.-W. Lee, T.-J. Oh, C. E. Misch, J. Shotwell, and H.L. Wang, "Effect of absorbable membranes on sandwich bone augmentation," Clinical Oral Implants Research, vol. 19, no. 1, pp. 32-41, 2008.

[12] O. T. Jensen, "Alveolar segmental "sandwich" osteotomies for posterior edentulous mandibular sites for dental implants," Journal of Oral and Maxillofacial Surgery, vol. 64, no. 3, pp. 471475, 2006.

[13] C. Marchetti, S. Trasarti, G. Corinaldesi, and P. Felice, "Interpositional bone grafts in the posterior mandibular region: a report on six patients," The International Journal of Periodontics and Restorative Dentistry, vol. 27, no. 6, pp. 547-555, 2007.
[14] A. Bianchi, P. Felice, G. Lizio, and C. Marchetti, "Alveolar distraction osteogenesis versus inlay bone grafting in posterior mandibular atrophy: a prospective study," Oral Surgery, Oral Medicine, Oral Pathology, Oral Radiology and Endodontology, vol. 105, no. 3, pp. 282-292, 2008.

[15] P. Felice, G. Cannizzaro, V. Checchi et al., "Vertical bone augmentation versus 7-mm-long implants in posterior atrophic mandibles. Results of a randomised controlled clinical trial of up to 4 months after loading," European journal of oral implantology, vol. 2, no. 1, pp. 7-20, 2009.

[16] A. Laviv, O. T. Jensn, E. Tarazi, and N. Casap, "Alveolar sandwich osteotomy in resorbed alveolar ridge for dental implants: a 4 year prospective study," Journal of Oral and Maxillofacial Surgery, vol. 72, pp. 292-303, 2014.

[17] M. S. Tonetti and C. H. F. Hämmerle, "Advances in bone augmentation to enable dental implant placement: consensus report of the sixth european workshop on periodontology," Journal of Clinical Periodontology, vol. 35, no. 8, pp. 168-172, 2008.

[18] P. Felice, C. Marchetti, A. Piattelli et al., "Vertical ridge augmentation of the atrophic posterior mandible with interpositional block grafts: bone from the iliac crest versus bovine anorganic bone," European Journal of Oral Implantology, vol. 1, no. 3, pp. 183-198, 2008.

[19] B. Fang, Y.-Z. Wan, T.-T. Tang, C. Gao, and K.-R. Dai, "Proliferation and osteoblastic differentiation of human bone marrow stromal cells on hydroxyapatite/bacterial cellulose nanocomposite scaffolds," Tissue Engineering A, vol. 15, no. 5, pp. 10911098, 2009.

[20] Y. Zhang and M. Zhang, "Synthesis and characterization of macroporous chitosan/calcium phosphate composite scaffolds for tissue engineering," Journal Biomedical Material Research, vol. 55, pp. 304-312, 2001.

[21] H. M. Hashemi and B. Javidi, "Comparison between interpositional bone grafting and osteogenic alveolar distraction in alveolar bone reconstruction," Journal of Oral and Maxillofacial Surgery, vol. 68, no. 8, pp. 1853-1858, 2010.

[22] M. Politi and M. Robiony, "Localized alveolar sandwich osteotomy for vertical augmentation of the anterior maxilla," Journal of Oral and Maxillofacial Surgery, vol. 57, no. 11, pp. 13801382, 1999.

[23] O. T. Jensen, L. Kuhlke, J.-F. Bedard, and D. White, "Alveolar segmental sandwich osteotomy for anterior maxillary vertical augmentation prior to implant placement," Journal of Oral and Maxillofacial Surgery, vol. 64, no. 2, pp. 290-296, 2006.

[24] K.-H. Bormann, M. M. Suarez-Cunqueiro, C. von See et al., "Forty sandwich osteotomies in atrophic mandibles: a retrospective study," Journal of Oral and Maxillofacial Surgery, vol. 69, no. 6, pp. 1562-1570, 2011.

[25] J. A. Elo, A. S. Herford, and P. J. Boyne, "Implant success in distracted bone versus autogenous bone-grafted sites," The Journal of Oral Implantology, vol. 35, no. 4, pp. 181-184, 2009. 

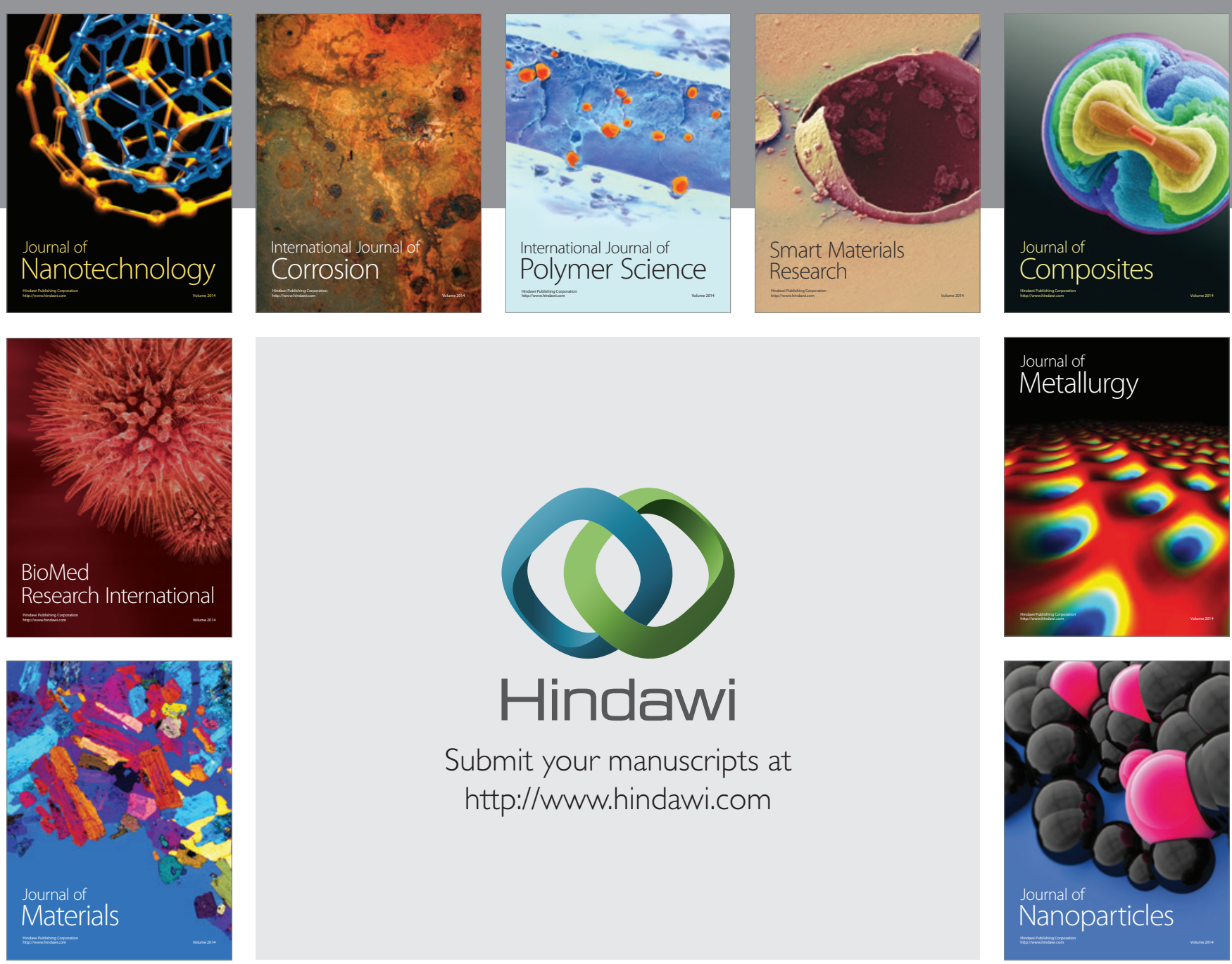

Submit your manuscripts at http://www.hindawi.com
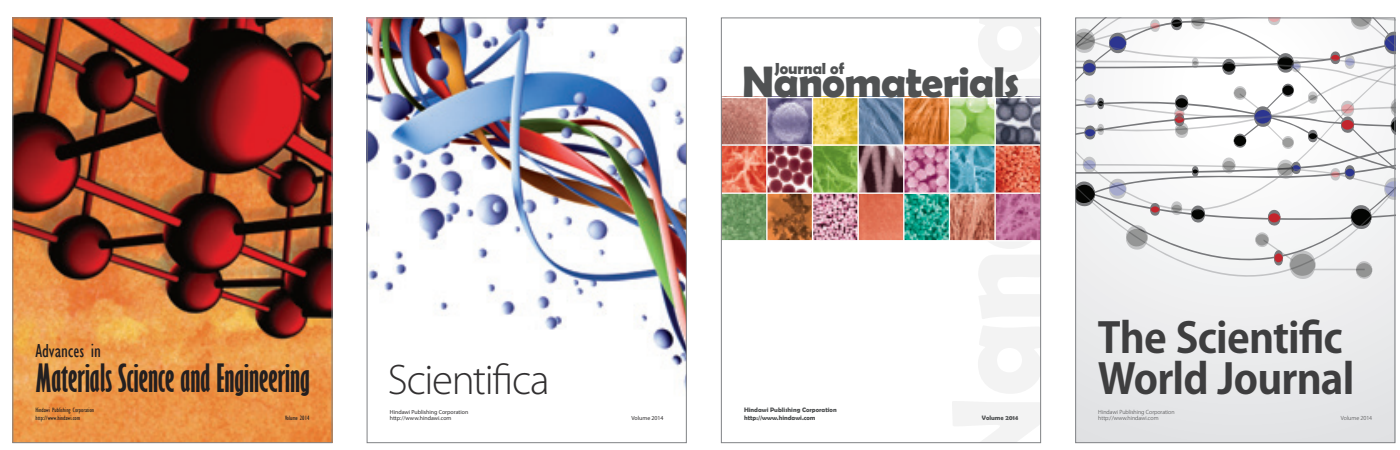

\section{The Scientific World Journal}
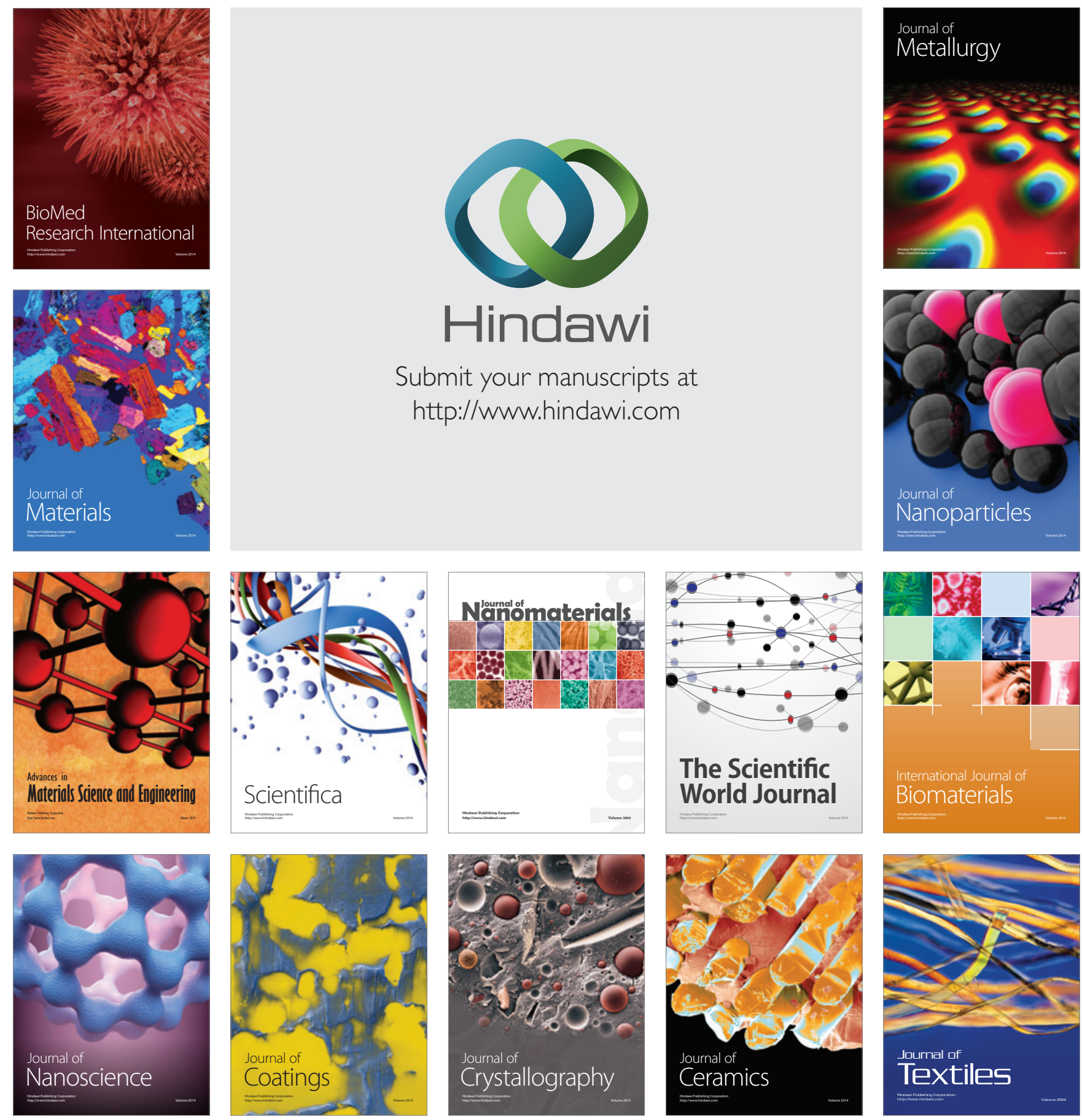